\title{
BMJ Open Associations of presence or absence of exercise and/or physical activity with non-restorative sleep by gender and age: a cross-sectional study
}

\author{
Tomoo Hidaka, ${ }^{\odot}$ Shota Endo, Hideaki Kasuga, Yusuke Masuishi, \\ Takeyasu Kakamu, ${ }^{\odot}$ Tomohiro Kumagai, Tetsuhito Fukushima
}

To cite: Hidaka T, Endo S, Kasuga $\mathrm{H}$, et al. Associations of presence or absence of exercise and/or physical activity with non-restorative sleep by gender and age: a crosssectional study. BMJ Open 2019;9:e025730. doi:10.1136/ bmjopen-2018-025730

- Prepublication history for this paper is available online. To view these files, please visit the journal online (http://dx.doi org/10.1136/bmjopen-2018025730).

Received 30 July 2018 Revised 21 February 2019 Accepted 1 April 2019
Check for updates

(C) Author(s) (or their employer(s)) 2019. Re-use permitted under CC BY-NC. No commercial re-use. See rights and permissions. Published by BMJ.

Department of Hygiene and Preventive Medicine, Fukushima Medical University, Fukushima, Japan

Correspondence to

Dr. Tomoo Hidaka;

thidaka@fmu.ac.jp

\section{ABSTRACT}

Objectives Non-restorative sleep (NRS) is related to qualitative aspects of sleep. The associations of NRS with exercise (EX; a planned and purposeful activity) and physical activity (PA; daily bodily movement) by gender and age have not yet been clarified. We investigated the associations of EX and/or PA with NRS by gender and age. Design A cross-sectional study.

Setting The data on gender, age, presence of NRS and engagement in EX and/or PA were obtained from database and questionnaire of specified medical check-ups in FY 2013 in Japan. The analysis was conducted in 2017. Participants The subjects comprised 90122 residents (38603 males and 51519 females), aged 40-74 years, who had completed the specified medical check-ups. Outcome measure The presence of NRS was assessed using a question asking whether or not the subjects usually got enough sleep. NRS was considered to be present when the subjects answered ' $N o$ '. Binary logistic regression analysis was used to assess the associations of presence or absence of EX and/or PA with NRS. The OR and $95 \% \mathrm{Cl}$ of NRS prevalence were calculated and compared between those engaged in both $\mathrm{EX}$ and $\mathrm{PA}$ and the others.

Results Except for 40s and 70s among males and 40s and 50s among females, the absence of EX or PA was associated with higher ORs of NRS than referent. ORs were more than twice compared with the referents in males in their 50s (OR 2.030(95\% Cl 1.675 to 2.459)) and 60s (OR $2.148(95 \% \mathrm{Cl} 1.970$ to 2.343$))$ and females in their $60 \mathrm{~s}$ ( $0 \mathrm{R} 2.142(95 \% \mathrm{Cl} 1.994$ to 2.302)) when they engaged in neither EX nor PA.

Conclusions Healthcare providers must take into account the similarities and differences in the associations of EX and/or PA with NRS by gender and age when they support people with NRS.

\section{INTRODUCTION}

Non-restorative sleep (NRS), a growing health problem in many countries, is defined as a subjective feeling of lack of refreshment on awakening and is related to qualitative aspects of sleep. ${ }^{1}$ Past studies demonstrated that NRS is associated with other various

\section{Strengths and limitations of this study}

- This study provides highly representative samples using data from a nationwide insurance organisation compared with previous studies.

- Unlike previous studies, in which associations of exercise $(\mathrm{EX})$ and physical activity (PA) with non-restorative sleep (NRS) were investigated separately, we categorised the presence or absence of EX and/ or PA into four patterns to determine their potential interaction and used them as independent variables for NRS.

- The major limitations of this study were that the degree and period of NRS were not revealed, and that the distinction between NRS and other sleep disorders was not fully clear since the information regarding NRS was collected by a single question with yes/no response.

health problems such as obesity, ${ }^{2}$ heart disease, ${ }^{3}{ }^{4}$ respiratory diseases, ${ }^{5}$ depressive symptoms ${ }^{6}$ and suicide. ${ }^{7}$ The prevalence of NRS in the general population is estimated to be approximately $10 \% .^{8}$

Past studies proved that NRS is associated with psychosocial factors related to ageing (eg, retirement or inactivity).${ }^{910}$ Similar to the findings of these studies, Myllyntausta et al reported decreased NRS prevalence in people aged 6-64 years compared with those aged 65 years or older, using longitudinal data of workers. ${ }^{11}$ Moreover, it was repeatedly confirmed that NRS is significantly more prevalent in women than in men. ${ }^{12} 13$ These past studies suggest that inevitable factors such as ageing and gender play a key role in the development of NRS.

Previous meta-analyses and systematic reviews concluded that both exercise (EX) and physical activity (PA) can contribute to the improvement of sleep disturbances. ${ }^{14}{ }^{15}$ Here, ' $\mathrm{EX}$ ' is defined as a planned and purposeful activity, while 'PA' is daily bodily movement. ${ }^{16}$ 
However, the associations of NRS with EX and PA have not yet been clarified. Importantly, NRS can be substantially differentiated from other sleep disturbances, such as insomnia, ${ }^{5} 17$ and its associations with $\mathrm{EX}$ and/or PA remain to be elucidated.

In the present study, we reveal for the first time the associations of the presence or absence of EX and/or PA with NRS according to gender and age.

\section{METHODS}

\section{Study design and participants}

In this cross-sectional study, the subjects comprised 92279 residents, aged 40- 74 years, who had undergone a specified medical check-up in FY 2013 in Fukushima Prefecture, Japan, by the National Health Insurance Organisation (NHIO). The specified medical check-up included a questionnaire asking about sleep quality (NRS). Together with the check-up data, the responses were recorded in the NHIO database. Individuals whose data on age, gender, habit of EX and PA and sleep quality were incomplete were excluded from the present study. The analysis was conducted in 2017, and the total number of participants was 90122 (38 603 males and 51519 females).

\section{Measurements}

EX and PA

The habit of EX and PA, including frequency, intensity and duration, was assessed using the following two respective questions used in the past studies ${ }^{18} 19$ with 'yes-no' responses: for EX, we asked 'Have you been exercising regularly for over $30 \mathrm{~min}$ at a time, during which you sweat lightly, two or more times weekly, for over a year?'; and for PA, we asked 'In your daily life do you walk or do equivalent PA for more than one hour a day?'

\section{Sleep quality (NRS)}

The presence of NRS was assessed using the following question, which was also used in a previous study, ${ }^{20}$ with 'yes-no' responses: 'Do you sleep well and enough?' NRS was considered to be present when the subjects answered 'No'.

\section{Statistical analysis}

The subjects were stratified according to gender and age (40-49, 50-59, 60-69 and 70 years or older), then analysed. Values were described as mean with SD or frequency, and the associations between the values were analysed by $\chi^{2}$ test and residual analysis. In residual analysis after $\chi^{2}$ test, the cells were regarded to have significantly more people than expected when the adjusted standardised residual values were greater than 1.96 , whereas the cells were regarded to have significantly fewer people than expected when the values were lower than -1.96 .

Binary logistic regression analysis was used to assess the associations of presence or absence of EX and/or PA with NRS, with those engaged in both EX and PA categorised

\begin{tabular}{|c|c|c|c|}
\hline & $\begin{array}{l}\text { Total } \\
(\mathrm{n}=90122)\end{array}$ & $\begin{array}{l}\text { Male } \\
(n=38603)\end{array}$ & $\begin{array}{l}\text { Female } \\
(n=51519)\end{array}$ \\
\hline Age (SD) & $64.3(6.9)$ & $64.4(7.1)$ & $64.3(6.8)$ \\
\hline \multicolumn{4}{|c|}{ Age group (years, \%) } \\
\hline $40-49$ & $4724(5.2)$ & $2220(5.8)$ & $2504(4.9)$ \\
\hline $50-59$ & $11738(13.0)$ & 4879 (12.6) & $6859(13.3)$ \\
\hline $60-69$ & $50856(56.4)$ & $21311(55.2)$ & $29545(57.3)$ \\
\hline$\geq 70$ & $22804(25.3)$ & $10193(26.4)$ & $12611(24.5)$ \\
\hline \multicolumn{4}{|c|}{ Engagement in EX or PA } \\
\hline Both & $23924(26.5)$ & $11569(30.0)$ & $12355(24.0)$ \\
\hline Only EX & $10493(11.6)$ & $4148(10.7)$ & $6345(12.3)$ \\
\hline Only PA & $13130(14.6)$ & $5514(14.3)$ & $7616(14.8)$ \\
\hline Neither & $42575(47.2)$ & $17372(45.0)$ & $25203(48.9)$ \\
\hline \multicolumn{4}{|l|}{ NRS } \\
\hline Present & 21276 (23.6) & 7825 (20.3) & $13451(26.1)$ \\
\hline Absent & $68846(76.4)$ & 30778 (79.7) & 38068 (73.9) \\
\hline
\end{tabular}

Both, engaging in both EX and PA; Only EX, engaging only in EX; Only PA, engaging only in PA; Neither, engaging in neither EX nor PA.

EX, exercise; NRS, non-restorative sleep; PA, physical activity.

as the referents. OR and the $95 \% \mathrm{CI}$ of NRS prevalence, compared with the referents, were calculated. A p-value of $<0.05$ was regarded as statistically significant. The data were analysed by SPSS statistics V.24.

\section{Patient and public involvement}

The participants were not involved in the development of the research question, outcome measures, design, recruitment and conduct of this study.

\section{RESULTS}

The characteristics of the study subjects are shown in table 1 . The mean ages of total, male and female subjects were 64.3 (SD: 6.9), 64.4 (SD: 7.1 ) and 64.3 (SD: 6.8) years, respectively. The most populated age group was 60-69 years in the total subjects $(56.4 \%)$, males $(55.2 \%)$ and females (57.3\%). Regarding EX and/or PA, the absence of both EX and PA was most common in total, male and female subjects $(47.2 \%, 45.0 \%$ and $48.9 \%$, respectively). NRS was present in $23.6 \%, 20.3 \%$ and $26.1 \%$ of the total, male and female subjects, respectively.

The associations of NRS with the presence or absence of EX and/or PA and age are shown in table 2. The $\chi^{2}$ test revealed that the presence or absence of EX and/or PA was significantly associated with the presence of NRS in both males and females ( $\mathrm{p}<0.001$ for both). Residual analysis showed that a significantly high number of males $(25.5 \%)$ and females $(31.6 \%)$ who did not engage in EX or PA had NRS. Regarding the associations of NRS and age, the $\chi^{2}$ test revealed that age was significantly associated with the presence of NRS in both males and 
Table 2 Associations of NRS with the presence or absence of EX and/or PA and age

\begin{tabular}{|c|c|c|c|c|}
\hline & \multicolumn{2}{|c|}{ Presence of NRS in male (\%) } & \multicolumn{2}{|c|}{ Presence of NRS in female (\%) } \\
\hline & Present & $P$ value & Present & $P$ value \\
\hline Engagement in EX and PA & & $<0.001$ & & $<0.001$ \\
\hline Only EX & $787(19.0)^{*}$ & & $1465(23.1)^{\star}$ & \\
\hline Only PA & $1030(18.7)^{\star}$ & & $1865(24.5)^{\star}$ & \\
\hline Age group (years, \%) & & $<0.001$ & & $<0.001$ \\
\hline $40-49$ & $639(28.8) \dagger$ & & $920(36.7) \dagger$ & \\
\hline $50-59$ & $1325(27.2) \dagger$ & & $2283(33.3) \dagger$ & \\
\hline $60-69$ & $4195(19.7)^{\star}$ & & $7527(25.5)^{*}$ & \\
\hline 70 or older & $1666(16.3)^{\star}$ & & $2721(21.6)^{\star}$ & \\
\hline
\end{tabular}

Boldface indicates statistical significance $(p<0.05)$ by chi-square test.

Both, engaging in both EX and PA; Only EX, engaging only in EX; Only P A, engaging only in PA; Neither, engaging in neither EX nor PA.

${ }^{*}$ Adjusted standardised residual $<-1.96$.

†Adjusted standardised residual $>1.96$.

EX, exercise; NRS, non-restorative sleep; PA, physical activity.

females ( $<<0.001$ for both). Residual analysis showed that a significantly high number of subjects in the 40-49 and 50-59 years age groups had NRS, in both males $(28.8 \%$ and $27.2 \%$, respectively) and females (36.7\% and $33.3 \%$, respectively).

The presence or absence of EX and/or PA by age is shown in table 3 . The $\chi^{2}$ test revealed that age was significantly associated with the presence or absence of EX and/or PA in both males and females ( $<<0.001$ for both). Residual analysis showed that a significantly high number of subjects fell in the following categories: subjects of both genders aged $\geq 70$ years engaging in both EX and PA ( $40.6 \%$ and $33.4 \%$, respectively); subjects of both genders aged $60-69$ and $\geq 70$ years engaging only in $\mathrm{EX}(11.1 \%$ and $11.5 \%$, respectively, for males; $13.3 \%$ and $13.6 \%$, respectively, for females); subjects of both genders aged 40-49 and $50-59$ years engaging only in PA $(16.7 \%$ and $15.8 \%$, respectively, for males; $17.9 \%$ and $16.4 \%$, respectively, for females); males aged 40-49, 50-59 and 60-69 years and females aged 40-49 and 50-59 years engaging neither in EX nor PA $(58.9 \%, 58.1 \%$ and $45.6 \%$, respectively for males; $66.5 \%$ and $61.9 \%$, respectively for females).

Table 3 Associations of presence or absence of EX and/or PA with age

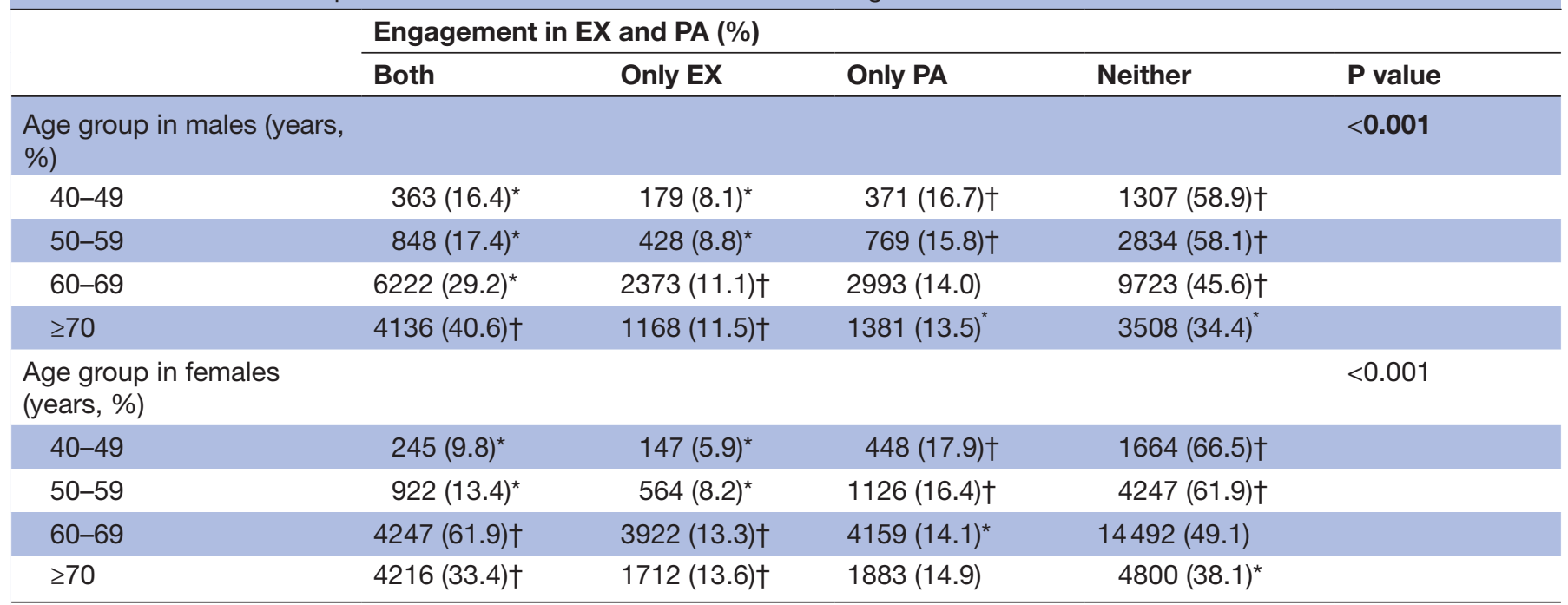

Boldface indicates statistical significance $(p<0.05)$ by chi-square test.

Both, engaging in both EX and PA; Only Ex, engaging only in EX; Only PA, engaging only in PA; Neither, engaging in neither EX nor PA.

*Adjusted standardised residual $<-1.96$.

†Adjusted standardised residual $>1.96$.

EX, e xercise; PA, physical activity. 
The binomial logistic regression model for the associations between NRS and the presence or absence of EX and/or PA is shown in table 4. Among males, the ORs were significantly higher than the referents in the 40-49years age group when engaged only in PA or neither (OR 1.536 (95\% CI 1.094 to 2.157), OR 1.787 (95\% CI 1.351 to 2.363 ), respectively) and in the $\geq 70$ years age group when engaged only in EX or neither (OR 1.491 (95\% CI 1.251 to 1.778 ), OR 1.864 (95\% CI 1.649 to 2.106), respectively). In the 50-59 and 60-69 years age groups, the ORs of NRS were significantly higher than the referents regardless of the presence or absence of EX and/or PA.

Among females, the ORs were significantly higher than the referents in the 40-49years age group when not engaged in EX or PA (OR 1.690 (95\% CI 1.256 to 2.273)) and in the 50 to 59 years age group when engaged only in PA or neither (OR 1.295 (95\% CI 1.063 to 1.578 ), OR 1.835 (95\% CI 1.560 to 2.159 ), respectively). In the 60-69 and $\geq 70$ years age groups, the ORs of NRS were significantly higher than the referents regardless of the presence or absence of EX and/or PA.

The ORs of both males and females in all age groups were significantly higher than the referents when they did not engage in EX nor PA.

\section{DISCUSSION}

The present study shows the associations between NRS prevalence and the presence or absence of EX and/or PA by gender and age group. It is important to note that the subjects were at the highest risk of NRS when they engaged in neither EX nor PA. The results of this study suggest that lifestyle interventions such as EX and PA may contribute to NRS prevention.

Regarding the descriptive statistics, approximately half of the female subjects reported that they did not engage in EX or PA. In line with a previous study indicating that females generally did not have a habit of EX compared with males, the females of the present study may have been passive about engaging in EX. ${ }^{21}$ Gender difference in the presence of NRS was also observed in the current study. Slightly more than one-fourth of the females had NRS, whereas NRS among the males was approximately one-fifth. The NRS prevalence of the Japanese females in the current study was higher than that of females in other countries, as reported by previous studies. While Ohayon et al estimated the general prevalence of NRS among females to be approximately $13 \%$, using data from countries such as Spain, Portugal, Finland, Italy, France, Germany, and the UK, ${ }^{8}$ other researchers have reported varying prevalence in other countries, such as approximately $6 \%$ in USA, ${ }^{5} 7 \%$ in Hong Kong ${ }^{22}$ and $21 \%$ in Sweden. ${ }^{12}$ Given the findings of these studies, it can be assumed that Japanese females are at higher risk of NRS.

Our analysis, using chi-square test stratified by gender, revealed that the presence of NRS was associated with the absence of both EX and PA. In this study, NRS was prevalent in the relatively younger age groups (40-49

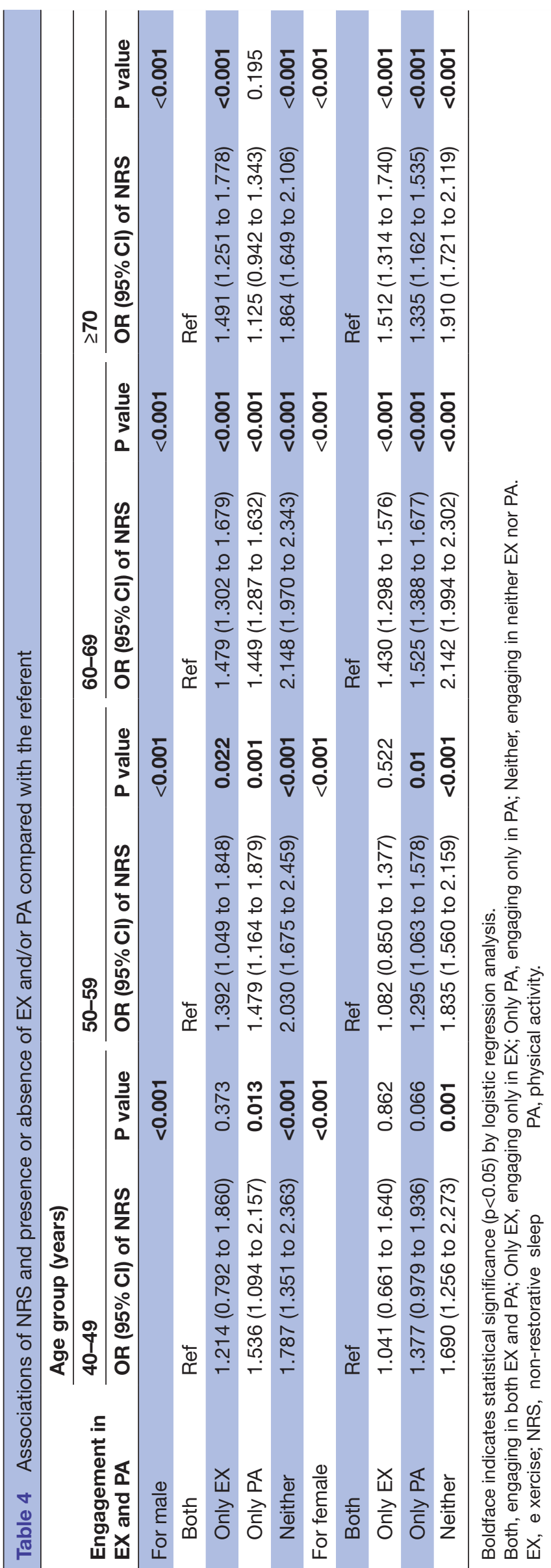


and $50-59$ years). As NRS is related to qualitative aspects of sleep, ${ }^{1}$ this finding was inconsistent with past studies, which have reported that sleep quality deteriorates with age. ${ }^{23}{ }^{24}$ Moreover, high numbers of subjects in these age groups answered that they engaged in neither EX nor PA (males $58.9 \%$ ( $40-49$ years) and $58.1 \%$ (50-59 years); females $66.5 \%$ ( $40-49$ years) and $61.9 \%$ (50-59 years) ). In light of these results, we believe that the risk of NRS may increase in people in their 40 s or 50 s who do not engage in EX or PA.

The binary logistic regression analysis in the current study revealed that the associations of presence or absence of EX and PA with NRS varied by gender and age. Among males aged 40-49 years, statistical differences were found between the referents and subjects who engaged either only in PA or neither, whereas there was no statistical difference between the referents and subjects who engaged only in EX. As mentioned previously, EX differs from PA in that it is a planned and purposeful activity, and EX is also known to be associated with sleep quality. ${ }^{25}$ The participants of $40 \mathrm{~s}$ males who could engage in EX might have been excelled at managing their own health including prevention of NRS. Japanese national survey as of 2017 indicated that the proportion of males having exercise habits was lower among 40 s $(24.4 \%)$ compared with other age groups such as 50 s, 60 s and 70 s $\left(27.1 \%, 42.9 \%\right.$ and $45.8 \%$, respectively).${ }^{26}$ In light of the fact that the engagement in EX is not popular among 40s males, the 40s among males engaging in EX may have been the specific group comprised of particularly health-conscious persons, and there is a possibility that such health consciousness may be associated with self-management of sleep quality and the absence of NRS. It is assumed that this interpretation is supported by the result that the proportion of 40s among males engaging EX in the present study (total proportion of persons engaged in both EX and PA and only in EX, 24.5\%) was approximately the same as that of above-mentioned national survey.

In males aged 50-59 and 60-69 years, there were significant differences in the prevalence of NRS between the referents and the subjects regardless of the presence or absence of EX and/or PA. As ORs were more than doubled compared with the referents when the subjects engaged in neither EX nor PA, the absence of both EX and PA may increase the risk of NRS, particularly in males aged 50-69 years. Our result that significantly high number of subjects in the 50-59 and 60-69year groups engaged in neither EX nor PA suggests that these age groups are at especially high risk of NRS. Workers aged 50-59 and 60-69 years may not have enough time for EX. For EX and/or PA that can be performed within a limited amount of time, the use of online management system ${ }^{27}$ and/or park management for sport ${ }^{28}$ may be effective for these groups. In males in their 70 s, there was no statistical difference in the prevalence of NRS between those engaged only in PA and the referents in the present study. For this age group, engagement in PA only and both EX and PA may be similarly associated with NRS risk. It may be more effective or enough only to increase PA in their daily life, rather than engaging in both EX and PA. It is important to pay attention to the mental status so as to promote PA among elderly people, considering that the level of PA often diminishes with age due to increased fear of injury, pain and falls, as reported by a previous study. ${ }^{29}$ Healthcare providers should keep in mind that the mental aspects can be a barrier to PA.

In the current study, no significant differences were observed between the female 40-49years group and the referents regarding the prevalence of NRS when they engaged in either EX or PA. It should be noted that the sample sizes were relatively small and $p$ value of the category engaged only in PA was nearly significant. The associations between the engagement only in PA and increased NRS risk may be revealed when the sample size is enlarged. To reduce the risk of NRS among this age group, it may be more realistic to engage only in EX orin both EX and PA.

In females aged 50-59 years, there was no significant difference in the prevalence of NRS from the referents when they engaged in EX only. This suggests that engagement only in EX can be fully effective when reducing NRS risk. Xi et al reported on health education effective for the establishment of a healthy lifestyle among females aged in their 40s and 50s and revealed that a carefully monitored diet had an important role in EX habit formation. ${ }^{30}$ Given this knowledge, monitoring one's diet may be effective for reducing NRS risk since it may contribute to the increase of engagement in EX.

In females in their 60 s and 70 s, we observed statistical differences in the prevalence of NRS between the referents and subjects, regardless of the presence or absence of EX and/or PA. Females in their 70s who engaged only in PA had lower OR than those engaged only in EX or neither. A previous systematic review concluded that participation in PA generally decreases with age, and that this tendency is particularly pronounced among elderly females (after age 60 years).$^{31}$ Moreover, $\mathrm{PA}$ is an important contributor to total energy expenditure in females overall. ${ }^{32}$ Another previous study suggested that social support is a critical factor related to participation in PA among females aged 65 years or older. ${ }^{33}$ The results of these studies indicate that the provision of social support may promote participation in PA among elderly women and may contribute to reducing the risk of NRS.

Limitations of this study were that the degree and period of NRS were not revealed and the distinction between NRS and other sleep disorders was not fully clear since the information regarding NRS was collected by a single question with yes/no response. In addition to these limitations related to the evaluation of NRS, the detail of EX and PA should be measured in terms of its total duration, intensity and amount. In particular, for the precise measurement of PA, the question item should include the recall period. Moreover, future study is required to analyse the associations between EX/PA and NRS using information regarding coexisting diseases such as obesity, diabetes mellitus, hypertension and dyslipidemia because such information may be potential confounding factors of the associations. In future 
study, it may be required to collect the information about psychological status possibly associated with NRS such as life stress to deeply interpret the high proportion of NRS in males and females in their 40s.

\section{CONCLUSIONS}

We revealed the associations of presence or absence of EX and/or PA with the prevalence of NRS by gender and age. The prevalence of NRS was higher when the subjects engaged in neither EX nor PA, compared with the referents who engaged in both EX and PA. Moreover, our results suggest that engaging in EX only among males in their 40s and females in their 40s and 50s and engaging in PA only among females in their 40s and males in their 70 s could be fully effective for reducing NRS risk. Healthcare providers must take into account the similarities and differences of associations of EX and/or PA with NRS by gender and age when they support people with NRS.

Acknowledgements We are grateful to NHIO for their assistance with data acquisition.

Contributors TH created the study concept and design, analysed and interpreted the data, drafted the initial manuscript and critically revised the manuscript for important intellectual content. SE, YM, TKu and HK provided technical support and critically revised the manuscript for important intellectual content. TKa conceptualised the study design. TF provided administrative support, acquired and interpreted the data and critically revised the manuscript for important intellectual content.

Funding The authors have not declared a specific grant for this research from any funding agency in the public, commercial or not-for-profit sectors.

Competing interests None declared.

Patient consent for publication Not required.

Ethics approval The study protocol was approved by the Ethics Committee of Fukushima Medical University, Fukushima, Japan (Application No. 2974).

Provenance and peer review Not commissioned; externally peer reviewed.

Data sharing statement Data available from the Dryad Digital Repository: https:// doi.org/10.5061/dryad.6f5k585.

Open access This is an open access article distributed in accordance with the Creative Commons Attribution Non Commercial (CC BY-NC 4.0) license, which permits others to distribute, remix, adapt, build upon this work non-commercially, and license their derivative works on different terms, provided the original work is properly cited, appropriate credit is given, any changes made indicated, and the use is non-commercial. See: http://creativecommons.org/licenses/by-nc/4.0/.

\section{REFERENCES}

1. Stone KC, Taylor DJ, McCrae CS, et al. Nonrestorative sleep. Sleep Med Rev 2008;12:275-88.

2. Resta O, Foschino Barbaro MP, Bonfitto P, et al. Low sleep quality and daytime sleepiness in obese patients without obstructive sleep apnoea syndrome. J Intern Med 2003;253:536-43.

3. Leineweber C, Kecklund G, Janszky I, et al. Poor sleep increases the prospective risk for recurrent events in middle-aged women with coronary disease. The Stockholm Female Coronary Risk Study. J Psychosom Res 2003;54:121-7.

4. Laugsand LE, Vatten LJ, Platou C, et al. Insomnia and the risk of acute myocardial infarction: a population study. Circulation 2011;124:2073-81.

5. Zhang J, Lamers F, Hickie IB, et al. Differentiating nonrestorative sleep from nocturnal insomnia symptoms: demographic, clinical, inflammatory, and functional correlates. Sleep 2013;36:671-9.
6. Sarsour K, Van Brunt DL, Johnston JA, et al. Associations of nonrestorative sleep with insomnia, depression, and daytime function. Sleep Med 2010;11:965-72.

7. Bernert RA, Turvey CL, Conwell Y, et al. Association of poor subjective sleep quality with risk for death by suicide during a 10year period: a longitudinal, population-based study of late life. JAMA Psychiatry 2014;71:1129-37.

8. Ohayon MM. Prevalence and correlates of nonrestorative sleep complaints. Arch Intern Med 2005;165:35-41.

9. Castro CM, Lee KA, Bliwise DL, et al. Sleep patterns and sleeprelated factors between caregiving and non-caregiving women. Behav Sleep Med 2009;7:164-79.

10. Morgan K. Daytime activity and risk factors for late-life insomnia. $J$ Sleep Res 2003;12:231-8.

11. Myllyntausta S, Salo P, Kronholm E, et al. Changes in sleep difficulties during the transition to statutory retirement. Sleep 2018;41.

12. Ohayon MM, Bader G. Prevalence and correlates of insomnia in the Swedish population aged 19-75 years. Sleep Med 2010;11:980-6.

13. Grandner MA, Petrov ME, Rattanaumpawan P, et al. Sleep symptoms, race/ethnicity, and socioeconomic position. J Clin Sleep Med 2013;9:897-905.

14. Driver HS, Taylor SR. Exercise and sleep. Sleep Med Rev 2000;4:387-402.

15. Chennaoui M, Arnal PJ, Sauvet F, et al. Sleep and exercise: a reciprocal issue?. Sleep Med Rev 2015;20:59-72.

16. World Health Organization. Physical activity. http://www.who.int/ dietphysicalactivity/pa/en/ (Accessed 10 Feb 2018).

17. Roth $T$, Zammit G, Lankford A, et al. Nonrestorative sleep as a distinct component of insomnia. Sleep 2010;33:449-58.

18. Hamano T, Fujisawa $Y$, Yamasaki M, et al. Contributions of social context to blood pressure: findings from a multilevel analysis of social capital and systolic blood pressure. Am J Hypertens 2011;24:643-6.

19. Wakasugi M, Kazama JJ, Yamamoto S, et al. A combination of healthy lifestyle factors is associated with a decreased incidence of chronic kidney disease: a population-based cohort study. Hypertens Res 2013;36:328-33.

20. Wakasugi M, Kazama JJ, Narita I, et al. Association between combined lifestyle factors and non-restorative sleep in Japan: a cross-sectional study based on a Japanese health database. PLoS One 2014;9:e108718.

21. Olson JS, Hummer RA, Harris KM. Gender and Health Behavior Clustering among U.S. Young Adults. Biodemography Soc Biol 2017;63:3-20.

22. Zhang J, Lam SP, Li SX, et al. The longitudinal course and impact of non-restorative sleep: a five-year community-based follow-up study. Sleep Med 2012;13:570-6.

23. Ohayon MM, Carskadon MA, Guilleminault C, et al. Meta-analysis of quantitative sleep parameters from childhood to old age in healthy individuals: developing normative sleep values across the human lifespan. Sleep 2004;27:1255-73.

24. Foley DJ, Monjan AA, Brown SL, et al. Sleep complaints among elderly persons: an epidemiologic study of three communities. Sleep 1995;18:425-32.

25. Sherrill DL, Kotchou K, Quan SF. Association of physical activity and human sleep disorders. Arch Intern Med 1998;158:1894-8.

26. Japanese Ministry of Health, Labour and Welfare. National Health and Nutrition Survey. 2017. https://www.mhlw.go.jp/content/ 10904750/000351576.pdf (Accessed 11 Dec 2018).

27. Hui SS, Xie YJ, Kwok RC, et al. Follow Your Virtual Trainer (FYVT): a randomised controlled trial protocol of IT-based lifestyle intervention programme to promote physical activity and health among middleaged Hong Kong Chinese. BMJ Open 2018;8:e017908.

28. Grunseit A, Richards J, Merom D. Running on a high: parkrun and personal well-being. BMC Public Health 2017;18:59.

29. Baert V, Gorus E, Mets T, et al. Motivators and barriers for physical activity in the oldest old: a systematic review. Ageing Res Rev 2011;10:464-74.

30. Xi S, Mao L, Chen X, et al. Effect of health education combining diet and exercise supervision in Chinese women with perimenopausal symptoms: a randomized controlled trial. Climacteric 2017;20:151-6.

31. Sun F, Norman IJ, While AE. Physical activity in older people: a systematic review. BMC Public Health 2013;13:449.

32. Jurj AL, Wen W, Gao YT, et al. Patterns and correlates of physical activity: a cross-sectional study in urban Chinese women. BMC Public Health 2007;7:213.

33. Plonczynski DJ. Physical activity determinants of older women: what influences activity? Medsurg Nurs 2003;12:213-59. 